\title{
Development of a model to analyze foot biomechanics using dynamic 3D surface scanning
}

\author{
-anonymous version-
}

Keywords: foot; 3D; scanning; modeling; kinematics

\section{Introduction}

The foot is a complex structure, bearing the responsibility for the load and propulsion of the entire human body during walking. Important variations in foot form and structure exist individually, and complaints of biomechanically related foot problems are a well-known issue. Appropriate analysis of the foot motion during a core activity like walking is required for the prevention, diagnosis and treatment of these foot problems.

The complex kinematics of the foot during roll-over are mostly studied by 2D video analysis or markerbased 3D motion analysis. However, these techniques are not able to gather full information about the 3D dynamic foot shape while walking. Detailed 3D data on the foot shape changes during roll-over contain essential information on foot function and behaviour which are difficult or not detectable with the current measurement techniques.

A recently developed dynamic 3D scanner (Vialux) is now able to scan the entire foot surface at $49 \mathrm{~Hz}$. In combination with force assessment, this innovative measurement technique has important potential in the field of biomechanics and motion analysis of the foot.

To accomplish the extraction of relevant parameters from the 3D dynamic surface data, an appropriate foot model has to be developed. Such a model has the capacity to result in kinematic variables concerning motion of the bones and joints, as well as superimposed shape variables induced by soft tissue deformation in the foot. The aim of this study is the extraction of such a foot model from a dynamic 3D surface scan.

\section{Methods}

The dynamic 3D foot scan system comprises 5 scanners, and foot analysis software developed by sportmedicin Tübingen [T. Schmeltzpfenning, 2009]. The measurement setup used for this study is similar, but works synchronized with a glass force plate to assess also kinetics during walking. The scanners are mounted around the scanning area in such a way that the entire foot surface is scanned (figure 1). This includes the plantar surface of the foot, assessed from underneath the glass force plate.



The system outputs a dynamic 3D point cloud of the geometry of the foot during the whole of the stance phase of gait. To retrieve relevant kinematic parameters from these data, an algorithm to calculate a multi-segment foot model is developed. This model aims to possess a high degree of individuality, allowing appropriate representations of all foot varieties.

In this multi-segment foot model, the amount, place and size of the segments were not predefined. Segments are calculated directly from the individual 3D dynamic point clouds. This is believed to be possible using an optical flow algorithm. The algorithm identifies which parts of the foot can be represented as rigid segments. It is a mathematical technique able to calculate the motion vector field between 2 subsequent positions. This vector field indicates which points move alike, and makes segment calculation possible (figure 2).

Characteristic to these segments is their dependence on the present motion. During a one-joint-motion, the foot will comprise a minor two segments. A footstep typically combines several movements though, resulting in several different constructions of segments.

Furthermore, the algorithm allows the extraction of other relevant kinematic parameters. Rotation axes (figure 2) give important information about the actions of the corresponding joints. Analogous to 
the segments, rotation axes are calculated individually and motion-specific.

a)

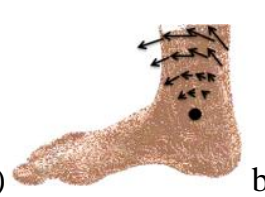

b)

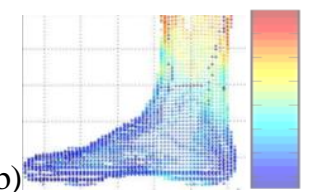

Figure 2: simplified example of a vector field, (a) direction and (b) norm with colour bar. Segments \& rotation axes can be extracted from these vectors.

\section{Results and Discussion}

As a proof of concept, foot motion vector fields in three stages of a footstep are calculated (figure 3a). For each of these stadia, the moving segments and their rotation axes can be found. The accuracy of these calculations is verified on the scan of an artificial foot with known axes and rigid segments (figure $3 b$ ). Performing the motion of rising to stand on the toes, the toe segment is successfully detected in the artificial foot. Axis direction is found with an accuracy of $11^{\circ}$.

a)
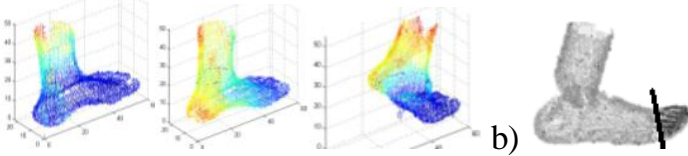

Figure 3: a) foot motion vector fields (norm) during midstance, heel off and toe off . b) artificial foot with detected toe rotation axis and toe segment

Dynamic 3D scanning is a novel alternative to analyse the foot, yet still expensive. To retrieve foot kinematics from these measurements, extraction of a segmented foot model based on the flow field has shown to be promising. The axis detection can still be improved, as these initial calculations were performed with a coarse quantization, and significant improvement is to be expected in refined calculations. Future work will apply improved segmentation and axis calculation algorithms on scanned human footsteps.

\section{Conclusions}

Retrieving relevant kinematic parameters from dynamic 3D surface scans is a process with broad possibilities in processing method. Development of a segmented foot model without predefined segments has advantages in its individual and motion-specific parameter extraction.

The development of the foot model is done in present studies, including validation and elaboration of its potential in foot research.

\section{References}

[1] Schmeltzpfenning T., Plank, C., Krauss I., Aswendt P., and Grau S. Dynamic foot scanning. A new approach for measurement of the human foot shape while walking. Footwear Science, 09, 28-30. [2] Vertommen H., Dewindt W., Bukenbergs S., Sevit R., and Peeraer L. The influence of different sports shoes on the pronation and pressure distribution under the foot. Congress Lecture 13th ISPO World Congress, 10, 659

\section{Acknowledgments}

Many thanks go to prof. J. Barron for his support using the optical flow implementation.

This work is supported by the Flemish agency for Innovation by Science and Technology (IWT and Era-Sme grant nr 100402). The authors would like to thank the whole Mobilab research team. Many thanks go also to prof. J. Barron for his support using the optical flow implementation.

*author: Inge Van den Herrewegen. Email: inge.van.den.herrewegen@khk.be 